\title{
Parameterization of Single Scattering Albedo (SSA) and Absorption Angstrom Exponent (AAE) with EC/OC for Aerosol Emissions from Biomass Burning
}

Rudra P. Pokhrel ${ }^{1}$, Nick L. Wagner ${ }^{2}$, Justin M. Langridge ${ }^{3}$, Daniel A. Lack ${ }^{4}$, Thilina Jayarathne ${ }^{5}$, Elizabeth A. Stone ${ }^{5}$, Chelsea E. Stockwell ${ }^{6}$, Robert J. Yokelson ${ }^{6}$ and Shane M. Murphy ${ }^{1}$

${ }^{1}$ Department of Atmospheric Science, University of Wyoming, Laramie, Wyoming, USA

${ }^{2}$ NOAA Earth System Research Laboratory, Chemical Sciences Division, Boulder, Colorado, USA

${ }^{3}$ Observation Based Research, Met Office, Fitzroy Road, Exeter, EX1 3PB, UK

${ }^{4}$ Transport Emissions, Air Quality and Climate Consulting, Brisbane, Australia

${ }^{5}$ Department of Chemistry, University of Iowa, Iowa City, Iowa, USA

${ }^{6}$ Department of Chemistry, University of Montana, Missoula, Montana, USA 
Table S1. Summary of fuels analyzed in this study and sampling locations.

\begin{tabular}{|l|l|l|l|l|}
\hline & $\begin{array}{l}\text { Stack } \\
\text { Furn }\end{array}$ & $\begin{array}{l}\text { Room } \\
\text { Burn }\end{array}$ & Fuel type & Sampling location \\
\hline $\begin{array}{l}\text { African grass } \\
\text { (tall) }\end{array}$ & 0 & 1 & Savana/Sourveld/Tall grass & Kruger National Park, R.S.A \\
\hline $\begin{array}{l}\text { African grass } \\
\text { (short) }\end{array}$ & 4 & 0 & Savana/Sourveld/Short grass & Kruger National Park, R.S.A \\
\hline Giant Cutgrass & 0 & 1 & Marsh & Jasper CO., SC \\
\hline Wiregrass & 0 & 1 & Pine forest understory & Chesterfield Co., SC \\
\hline Peat (CAN) & 2 & 0 & Boreal Peat & Ontario \& Alberta, Canada \\
\hline Peat (NC) & 2 & 1 & Temperate Peat & $\begin{array}{l}\text { Green Swamp \& Alligator River } \\
\text { NWR, NC }\end{array}$ \\
\hline Peat (IN) & 2 & 0 & Indonesian Peat & Central Kalimantan \\
\hline Organic Hay & 1 & 2 & Crop residue & Fort Collins, CO \\
\hline $\begin{array}{l}\text { Organic Wheat } \\
\text { Straw }\end{array}$ & 1 & 2 & Crop residue & Fort Collins, CO \\
\hline $\begin{array}{l}\text { Conv. Wheat } \\
\text { Straw }\end{array}$ & 0 & 1 & Crop residue & Walla Walla CO., WA \\
\hline Sugar Cane & 0 & 1 & Crop residue & Thibodaux, LA \\
\hline Rice Straw & 1 & 2 & Crop residue & CA, China, Malaysia, Taiwan \\
\hline Ponderosa Pine & 5 & 4 & Temperate Forest & Outskirts of Missoula, MT \\
\hline Black Spruce & 0 & 3 & Boreal Forest & South of Fairbanks, AK \\
\hline Chamise & 1 & 1 & Chaparral & San Jacinto Mountains, CA \\
\hline Manzanita & 1 & 1 & Chaparral & San Jacinto Mountains, CA \\
\hline Total & 20 & 21 & & \\
\hline & & & & \\
\hline
\end{tabular}


Table S2. SSA, AAE, MCE, EC to OC ratio, and mass of fuel burned for all fuels measured during stack and room burns of this work. S and $\mathrm{K}$ represent the short and tall African grass and the number after $\mathrm{S}$ or $\mathrm{K}$ indicate the collection plots. The \pm values after AAE are the one standard deviation of the slope and for EC/OC is the propagated error from $\mathrm{EC}$ and $\mathrm{OC}$ uncertainty.

\begin{tabular}{|c|c|c|c|c|c|c|c|c|}
\hline \multirow[b]{2}{*}{ ID } & \multirow[b]{2}{*}{ Fuel Type } & \multicolumn{3}{|c|}{ SSA } & \multirow[b]{2}{*}{ AAE } & \multirow[b]{2}{*}{ MCE } & \multirow[b]{2}{*}{ EC/OC } & \multirow[b]{2}{*}{ Mass (g) } \\
\hline & & 405 & 532 & 660 & & & & \\
\hline 9 & Manzanita & 0.79 & 0.8 & 0.79 & $2.05 \pm 0.37$ & 0.93 & NA & 270 \\
\hline 18 & Ponderosa Pine & 0.88 & 0.95 & 0.95 & $3.69 \pm 0.48$ & 0.918 & $0.061 \pm 0.019$ & 202 \\
\hline 20 & Chamise & 0.79 & 0.78 & 0.83 & $2.52 \pm 1.58$ & 0.913 & NA & 250 \\
\hline 32 & Rice Straw & 0.71 & 0.86 & 0.91 & $4.16 \pm 0.11$ & 0.943 & $0.109 \pm 0.028$ & 430 \\
\hline 61 & NC Peat & 0.94 & 1 & 1 & $10.43 \pm 1.11$ & 0.683 & ND & NA \\
\hline 62 & Ponderosa Pine & 0.83 & 0.91 & 0.89 & $2.85 \pm 0.48$ & 0.946 & $0.083 \pm 0.016$ & 4029 \\
\hline 70 & Ponderosa Pine & 0.93 & 0.99 & 0.99 & $5.95 \pm 0.79$ & 0.848 & $0.021 \pm 0.021$ & 274 \\
\hline 81 & African Grass S2 & 0.72 & 0.78 & 0.81 & $2.25 \pm 0.33$ & 0.97 & NA & 436 \\
\hline 82 & African Grass S1 & 0.53 & 0.55 & 0.54 & $1.92 \pm 0.27$ & 0.978 & NA & 416 \\
\hline 84 & Ponderosa Pine & 0.82 & 0.87 & 0.87 & $2.05 \pm 0.23$ & 0.925 & NA & 3860 \\
\hline 87 & Organic Hay & 0.81 & 0.91 & 0.94 & $3.46 \pm 0.08$ & 0.941 & NA & 412 \\
\hline 91 & African Grass S1 & 0.84 & 0.92 & 0.87 & $3.05 \pm 0.39$ & 0.97 & NA & 580 \\
\hline 92 & African Grass S2 & 0.58 & 0.62 & 0.58 & $1.72 \pm 0.08$ & 0.972 & NA & 455 \\
\hline 95 & Ponderosa Pine & 0.78 & 0.85 & 0.85 & $2.48 \pm 0.08$ & 0.933 & NA & 150 \\
\hline 96 & Organic Wheat & 0.73 & 0.76 & 0.87 & $3.43 \pm 1.13$ & 0.965 & $0.234 \pm 0.063$ & 154 \\
\hline 112 & Canadian Peat & 0.97 & 1 & 1 & $5.11 \pm 1.58$ & 0.811 & $0.011 \pm 0.015$ & NA \\
\hline 113 & NC Peat & 0.94 & 0.98 & 0.98 & $3.68 \pm 0.47$ & 0.692 & $0.008 \pm 0.008$ & NA \\
\hline 114 & Indonesian Peat & 0.93 & 1 & 0.99 & $7.44 \pm 3.27$ & 0.744 & $0.005 \pm 0.010$ & NA \\
\hline 124 & Canadian Peat & 0.91 & 0.99 & 0.99 & $6.78 \pm 0.26$ & 0.798 & NA & NA \\
\hline 125 & Indonesian Peat & 0.93 & 0.98 & 0.99 & $8.03 \pm 2.50$ & 0.872 & NA & NA \\
\hline 129 & $\begin{array}{l}\text { Ponderosa Pine } \\
\text { brown/green }\end{array}$ & 0.92 & 0.98 & 0.98 & $4.92 \pm 0.46$ & 0.839 & $0.067 \pm 0.018$ & 200 \\
\hline 130 & $\begin{array}{l}\text { California Rice } \\
\text { Straw }\end{array}$ & 0.75 & 0.84 & 0.84 & $2.98 \pm 0.20$ & 0.961 & $0.188 \pm 0.031$ & 147 \\
\hline 131 & Black Spruce & 0.87 & 0.93 & 0.93 & $2.98 \pm 0.05$ & 0.957 & $0.092 \pm 0.017$ & 639 \\
\hline 132 & Organic Wheat & 0.82 & 0.88 & 0.88 & $2.58 \pm 0.01$ & 0.956 & $0.124 \pm 0.024$ & 638 \\
\hline 133 & $\begin{array}{l}\text { Conventional } \\
\text { Wheat }\end{array}$ & 0.79 & 0.85 & 0.86 & $2.67 \pm 0.03$ & 0.956 & $0.147 \pm 0.022$ & 494 \\
\hline 134 & Black Spruce & 0.87 & 0.93 & 0.93 & $2.97 \pm 0.12$ & 0.957 & NA & 1077 \\
\hline 135 & Chamise & 0.58 & 0.61 & 0.54 & $1.95 \pm 0.06$ & 0.954 & NA & 667 \\
\hline 136 & Manzanita & 0.52 & 0.56 & 0.46 & $1.68 \pm 0.09$ & 0.959 & $0.694 \pm 0.059$ & 1064 \\
\hline 137 & Black Spruce & 0.82 & 0.9 & 0.84 & $2.32 \pm 0.70$ & 0.962 & NA & 1602 \\
\hline 138 & Organic Hay & 0.85 & 0.94 & 0.92 & $3.25 \pm 0.79$ & 0.95 & NA & 592.2 \\
\hline 140 & Ponderosa Pine & 0.88 & 0.94 & 0.91 & $2.21 \pm 0.61$ & 0.928 & $0.039 \pm 0.012$ & 1672 \\
\hline
\end{tabular}




\begin{tabular}{|l|l|l|l|l|l|l|l|l|}
141 & Wire Grass & 0.36 & 0.38 & 0.35 & $0.85 \pm 0.21$ & 0.969 & $1.351 \pm 0.112$ & 540 \\
\hline 142 & $\begin{array}{l}\text { Ponderosa Pine } \\
\text { brown/green }\end{array}$ & 0.79 & 0.83 & 0.82 & $2.19 \pm 0.19$ & 0.952 & NA & 1529 \\
\hline 143 & $\begin{array}{l}\text { California Rice } \\
\text { Straw }\end{array}$ & 0.87 & 0.95 & 0.97 & $4.48 \pm 0.05$ & 0.939 & NA & 902 \\
\hline 144 & Ponderosa Pine & 0.86 & 0.91 & 0.93 & $2.68 \pm 0.21$ & 0.927 & $0.037 \pm 0.013$ & 1731 \\
\hline 145 & African Grass K3 & 0.45 & 0.32 & 0.26 & $1.29 \pm 0.48$ & 0.975 & $1.176 \pm 0.096$ & 1078 \\
\hline 146 & Organic Hay & 0.85 & 0.94 & 0.96 & $3.55 \pm 0.08$ & 0.937 & $0.027 \pm 0.010$ & 1335 \\
\hline 147 & Sugarcane & 0.85 & 0.93 & 0.94 & $4.02 \pm 0.13$ & 0.934 & $0.05 \pm 0.020$ & 867 \\
\hline 148 & Giant Saw Grass & 0.39 & 0.43 & 0.44 & $1.83 \pm 0.34$ & 0.925 & $2.083 \pm 0.175$ & 2000 \\
\hline 149 & Organic Wheat & 0.77 & 0.83 & 0.83 & $2.58 \pm 0.08$ & 0.962 & $0.181 \pm 0.028$ & 393 \\
\hline 150 & $\begin{array}{l}\text { North Carolina } \\
\text { Peat }\end{array}$ & 0.95 & 0.99 & 1 & $6.25 \pm 0.63$ & 0.803 & NA & NA \\
\hline
\end{tabular}


Table S3. Same as Table 5 of main text but for $405 \mathrm{~nm}$.

\begin{tabular}{|l|l|l|l|r|l|l|}
\hline Biomass Types & MCE & BC/OC & $\begin{array}{l}\text { SSA_405 } \\
\text { MCE } \\
\text { Approach }\end{array}$ & $\begin{array}{l}\text { SSA_405 } \\
\text { EC/OC } \\
\text { Approach }\end{array}$ & $\begin{array}{l}\text { SSA_405 } \\
\text { EC/EC+OC) } \\
\text { Approach }\end{array}$ & $\begin{array}{l}\text { \% Difference } \\
\text { In Predicted } \\
\text { SSA }\end{array}$ \\
\hline Tropical Forest & 0.95 & 0.11 & 0.74 & 0.81 & 0.83 & $-9.46 /-12.16$ \\
\hline Savanna & 0.96 & 0.14 & 0.63 & 0.78 & 0.80 & $-23.81 /-26.98$ \\
\hline Crop Residue & 0.94 & 0.33 & 0.77 & 0.69 & 0.70 & $10.39 / 9.09$ \\
\hline Pasture Maintenance & 0.92 & 0.09 & 0.84 & 0.82 & 0.84 & $2.38 / 0$ \\
\hline Boreal Forest & 0.92 & - & 0.84 & & - & - \\
\hline Temperate Forest & 0.95 & - & 0.73 & & - & - \\
\hline Extra tropical Forest & 0.93 & 0.07 & 0.83 & 0.85 & 0.86 & $-2.41 /-3.61$ \\
\hline Peat land & 0.9 & 0.03 & 0.90 & 0.89 & 0.89 & $1.11 / 1.11$ \\
\hline Chaparral & 0.96 & 0.35 & 0.64 & 0.68 & 0.68 & $-6.25 /-6.25$ \\
\hline Open Cooking & 0.95 & 0.29 & 0.70 & 0.71 & 0.72 & $-1.43 /-2.86$ \\
\hline Patsari Stoves & 0.97 & 0.39 & 0.54 & 0.67 & 0.67 & $-24.07 /-24.07$ \\
\hline Charcoal Making & 0.86 & 0.03 & 0.93 & 0.89 & 0.89 & $4.30 / 4.30$ \\
\hline Charcoal Burning & 0.93 & 0.77 & 0.82 & 0.55 & 0.53 & $32.93 / 35.37$ \\
\hline Dung Burning & 0.89 & 0.29 & 0.90 & 0.7 & 0.71 & $22.22 / 21.11$ \\
\hline Garbage Burning & 0.97 & 0.12 & 0.54 & 0.8 & 0.82 & $-48.15 / 51.85$ \\
\hline
\end{tabular}

${ }^{1}$ Data from Akagi et al., 2011 